\title{
Activity-based Screening Assay for Mono-ADP-ribosylhydrolases
}

Sarah Wazir, Mirko M. Maksimainen, Heli I. Alanen, Albert Galera-Prat and Lari Lehtiö*

Faculty of Biochemistry and Molecular Medicine \& Biocenter Oulu, University of Oulu, Oulu, Finland

*Corresponding author email: lari.lehtio@,oulu.fi

Keywords: ADP-ribosylhydrolases, macrodomain, screening assay, activity assay 


\section{Abstract}

ADP-ribosylation is a post-translational modification involved in the regulation of many vital cellular processes. This post-translational modification is carried out by ADP-ribosyltransferases converting $\beta$ $\mathrm{NAD}^{+}$into nicotinamide and protein linked ADP-ribosyl group or a chain of poly-ADP-ribose. The reverse reaction, release of ADP-ribose from the acceptor molecule, is catalyzed by ADPribosylhydrolases. Several hydrolases contain a macrodomain fold and activities of human macrodomain protein modules vary from reading or erasing mono- and poly-ADP-ribosylation. Macrodomains have been linked to diseases, such as cancer, making them potential drug targets. Discovery of inhibitors requires robust biochemical tools mostly lacking for hydrolases and here we describe an inhibitor screening assay against mono-ADP-ribosylhydrolyzing enzymes. The activitybased assay uses an $\alpha-\mathrm{NAD}^{+}$, anomer of $\beta-\mathrm{NAD}^{+}$, which is accepted as a substrate by MacroD1, MacroD2 and ARH3 due to its resemblance to the protein linked ADP-ribose. The amount of $\alpha-\mathrm{NAD}^{+}$ present after hydrolysis is measured by chemically converting it on a microtiter plate to a fluorescent compound. We optimized the assay for MacroD2 and performed a proof-of-concept compound screening. Three compounds were identified as screening hits with $\mu \mathrm{M}$ potency. However, further characterization of the compounds identified them as protein destabilizers excluding further follow up studies. Validation and screening demonstrated the usability of the in vitro assay for MacroD2 and we also demonstrate the applicability of the assay as a tool for other human ADP-ribosylhydrolases. 


\section{Introduction}

$\mathrm{NAD}^{+}$dependent protein ADP-ribosylation is a reversible post-translational modification carried out mainly by ADP-ribosyltransferases (PARPs/ARTDs), attaching mono-ADP-ribose (MAR) or polyADP-ribose (PAR) to the target proteins, DNA or RNA ${ }^{1-4}$. The modification is used to regulate vital cellular processes for instance DNA repair, cell signaling and transcription. The ADP-ribosylation is read and/or erased mainly by Nudix hydrolases, macrodomains and ADP-ribosylhydrolases (ARHs) ${ }^{5,6}$. In human, there are 16 macrodomains, which are structurally conserved protein modules. However, they have distinct functions relating to ADP-ribosylation. Some of the macrodomains bind and some also hydrolyze MAR/PAR ${ }^{6,7}$. MacroD1 and MacroD2 act as erasers of MAR. In addition to MAR, they hydrolyze metabolites produced independently of ARTDs activity, for instance, O-acetyl-ADP-ribose generated by sirtuin family proteins ${ }^{6,8}$. Both enzymes as well as the other human macrodomains are strongly associated to human cancers where they are typically overexpressed. For example, in pancreatic cancer, the up-regulation of MacroD1 promotes proliferation, migration and invasion of cancer cells ${ }^{9}$ and overexpression of MacroD2 mediates estrogen independent growth and tamoxifen resistance in breast cancer ${ }^{10}$. MacroD2 also controls DNA repair signaling mediated by ARTD1/PARP1 as loss of MacroD2 has been seen to cause impairment of ARTD1/PARP1 activity leading to DNA repair deficiency of colorectal cancer cells ${ }^{11}$. 
In addition to MacroD1 and MacroD2, ARH1 and ARH3 from the family of ADP-ribosylhydrolases hydrolyze O-acetyl-ADP-ribose. Moreover, ARH1 and ARH3 also erase ADP-ribosylation, but the activity of ARH1 is very weak, 1\%, compared to ARH3. ARH3 is a main eraser removing Serine linked ADP-ribose ${ }^{12}$. The family also contains ARH2, which binds ADP-ribose but is not shown to be enzymatically active ${ }^{13,14}$. ARH1 and ARH3 have different roles in cells where ARH1 is involved in tumorigenesis and activities of bacterial toxins, whereas ARH3 contributes to the apoptosis pathway, called parthanatos, by degrading the PAR produced by ARTD1/PARP1 in response to oxidative stressinduced DNA damage ${ }^{15,16}$. 
Due to their roles as hydrolases, macrodomains and ARHs have been implicated to potential drug targets against cancer. To date, the inhibitors discovered for poly(ADP-ribose) glycohydrolase (PARG) and MacroD1 have indeed provided important information about the roles of the enzymes and also their potencies as drug targets. The recent inhibitor of PARG sensitizes cancer cells to radiationinduced DNA damage and stalls the replication fork hindering cancer cell survival ${ }^{17}$. In the case of MacroD1, the inhibitor (MRS2578) blocks MacroD1 interaction with the RNA-dependent kinase (PKR) that eventually sensitizes cancer cells to DNA damage ${ }^{18}$. These discoveries demonstrate well the growing need of small molecule compounds selectively inhibiting readers and erasers of ADPribosylation. However, no potent inhibitors are available for many human macrodomains and ARHs. One reason is a lack of inhibitor screening assays allowing identification of early hit compounds. In this paper, we describe a fluorescence based homogenous assay to screen for inhibitors against MAR hydrolases. The assay utilizes $\alpha-\mathrm{NAD}^{+}$, which mimics protein ADP-ribosylation due to the anomerization of $\beta-\mathrm{NAD}^{+}$. Indeed, several macrodomains have recently been shown to be able to hydrolyze $\alpha-\mathrm{NAD}^{+19}$ and despite that this does not measure removal of ADP-ribosyl group from a protein, it provides a way to measure hydrolysis activity of a substrate analogous to it. After enzymatic reaction, $\alpha-\mathrm{NAD}^{+}$is chemically converted to a fluorophore for quantification (Suppl. Fig. 1). We optimized the assay for MacroD2 and performed inhibitor screening against the enzyme. We also demonstrate that the assay is applicable for other erasers of ADP-ribosylation, such as MacroD1 and ARH3.

\section{Materials and Methods}

\section{Expression Constructs, Protein Expression and Purification}


MacroD1 (residues 58-325), MacroD2 (residues 7-243), PARP2 and TARG1 constructs were previously cloned in pNH-TrxT vector and proteins were produced and purified using established protocols $^{20,21}$.

The cDNA of full-length PARG was purchased from Source BioScience (UK) and the catalytic domain of PARG (residues 448-976) was cloned also into pNH-TrxT vector. PARG was expressed and purified as described earlier for other macrodomains ${ }^{21}$. Briefly, PARG construct was expressed in Escherichia coli Rosetta 2 (DE3) cells using terrific broth (TB) auto-induction media containing $8 \mathrm{~g} / \mathrm{L}$ glycerol and $100 \mu \mathrm{g} / \mathrm{mL}$ kanamycin and $37 \mu \mathrm{g} / \mathrm{mL}$ chloramphenicol. Cell suspension was supplemented by adding $0.1 \mathrm{mg} / \mathrm{mL}$ lysozyme, $0.02 \mathrm{mg} / \mathrm{mL}$ DNAase and $0.1 \mathrm{mM}$ pefabloc (protease inhibitor) (Sigma-Aldrich, USA). Cells were further lysed by sonication and the lysate was collected by centrifugation. The supernatant was filtered using $0.45 \mu \mathrm{m}$ filter and loaded onto a $5 \mathrm{~mL}$ HisTrap HP nickel-nitrilotriacetic acid (Ni-NTA) column (GE Healthcare). The column was washed with lysis buffer, followed by washed buffer containing $25 \mathrm{mM}$ imidazole and protein was eluted by increasing the imidazole concentration to $300 \mathrm{mM}$ in the buffer. Additional tag for protein was cleaved by using TEV protease at $+4{ }^{\circ} \mathrm{C}$ overnight followed by a reverse-Ni-affinity chromatography. The protein was finally purified with size exclusion chromatography using 16/600 Superdex 75 column (GE Healthcare) preequilibrated column with $50 \mathrm{mM}$ HEPES pH 7.5, $300 \mathrm{mM} \mathrm{NaCl}, 10 \%$ glycerol, $0.5 \mathrm{mM}$ TCEP and flash freezed in small aliquots in liquid $\mathrm{N}_{2}$ at $-70{ }^{\circ} \mathrm{C}$.

Linear, double-stranded DNA fragments (gBlocks) of full-length ARH1 and ARH2 were purchased from Integrated DNA Technologies (IDT). The gBlocks contained 5 ' and 3 ' overhangs for sequenceand ligation-independent cloning (SLIC), which was used to clone the constructs in pNIC-Bsa4. pET30-ARH3-His-Sumo-HA was a gift from Thomas Muir (Addgene plasmid \#11578) ${ }^{22}$. 
ARHs constructs were expressed in E. coli BL21 (DE3) cells using terrific broth (TB) auto-induction media containing $8 \mathrm{~g} / \mathrm{L}$ glycerol and $50 \mu \mathrm{g} / \mathrm{mL}$ kanamycin. The cells were allowed to grow at $37{ }^{\circ} \mathrm{C}$ until the optical density at $600 \mathrm{~nm}\left(\mathrm{OD}_{600}\right)$ reached 1 and the temperature was reduced to $18{ }^{\circ} \mathrm{C}$ or 15 ${ }^{\circ} \mathrm{C}$ in case of ARH3. The cells were collected by centrifugation and suspended in lysis buffer $50 \mathrm{mM}$ HEPES pH 7.5, $10 \mathrm{mM} \mathrm{MgCl}$, $300 \mathrm{mM} \mathrm{NaCl,} \mathrm{10 \%} \mathrm{glycerol,} 10 \mathrm{mM}$ imidazole, $0.5 \mathrm{mM}$ TCEP, $20 \mathrm{ug} /$ $\mathrm{mL}$ DNase and $0.1 \mathrm{mM}$ Pefabloc and stored at $-20^{\circ} \mathrm{C}$. The cells were lysed by sonication and the lysate was cleared by centrifugation. The supernatant was filtered using $0.45 \mu \mathrm{m}$ filter and loaded onto a $5 \mathrm{~mL}$ HiTrap Chelating HP column (GE Healthcare) pre-charged with nickel. The column was washed with lysis buffer, followed by wash buffer containing $25 \mathrm{mM}$ imidazole. His-Sumo tag from ARH3 was cleaved by using Senp 2 protease at $+4{ }^{\circ} \mathrm{C}$ overnight followed by a reverse-Ni-affinity chromatography. His-tags in ARH1 and ARH2 were resistant to cleavage by TEV protease at $+4{ }^{\circ} \mathrm{C}$ overnight and they were further purified by eluting from the second Ni-affinity column stepwise with increasing imidazole concentration between $40-250 \mathrm{mM}$. The purest protein fractions were finally purified with size exclusion chromatography using 16/600 Superdex 75 column pre-equilibrated with $30 \mathrm{mM}$ HEPES pH 7.5, $10 \mathrm{mM} \mathrm{MgCl}_{2}, 300 \mathrm{mM} \mathrm{NaCl}, 10 \%$ glycerol, $0.5 \mathrm{mM}$ TCEP and flash frozen in small aliquots in liquid $\mathrm{N}_{2}$ at $-70{ }^{\circ} \mathrm{C}$. Purity of the used proteins and thermal melting curves are shown in Suppl. Fig.

\section{$2 A \& 2 B$.}

AF1521 macrodomain was produced for detection of poly(ADP-ribose) hydrolysis. Expression was carried out in BL21(DE3) cells in TB auto-induction media containing $8 \mathrm{~g} / \mathrm{L}$ glycerol at $18^{\circ} \mathrm{C}$. The cells were harvested by centrifugation and suspended in lysis buffer $50 \mathrm{mM}$ HEPES pH 7.5, $500 \mathrm{mM} \mathrm{NaCl}$, 10\% glycerol, $10 \mathrm{mM}$ imidazole, $0.5 \mathrm{mM}$ TCEP and $0.1 \mathrm{mM}$ Pefabloc. Cell lysis was done by sonication and the lysate was cleared by centrifugation. The supernatant was loaded onto a $5 \mathrm{~mL}$ HiTrap Chelating HP column (GE Healthcare) pre-charged with nickel. The column was first washed 
with lysis buffer and then with wash buffer containing $25 \mathrm{mM}$ imidazole. Protein was then eluted with buffer containing $300 \mathrm{mM}$ imidazole. Eluted sample was further purified using GST-Trap column (GE Healthcare), washed using the same buffer and eluted in $50 \mathrm{mM}$ Tris pH 8.0, $200 \mathrm{mM} \mathrm{NaCl}, 0.5 \mathrm{mM}$ TCEP, $10 \mathrm{mM}$ reduced gluthatione. Sample was dialyzed against $30 \mathrm{mM}$ HEPES pH 7.5, $350 \mathrm{mM}$ $\mathrm{NaCl}, 10 \%$ glycerol, $0.5 \mathrm{mM}$ TCEP and stored at $-70^{\circ} \mathrm{C}$.

\section{Poly(ADP-ribose) hydrolysis assays}

PARG, TARG1, ARH1 and ARH3 hydrolytic activity was tested against PAR from auto-modified PARP2. For these assays, $7.6 \mu \mathrm{M}$ PARP2 was incubated with $1 \mathrm{mM} \mathrm{NAD}{ }^{+}$and activating oligo DNA for 2 hours at room temperature in $50 \mathrm{mM}$ Tris $\mathrm{pH} 8.0,5 \mathrm{mM} \mathrm{MgCl}$. Reaction was stopped by addition of SDS buffer and analysed by SDS-PAGE. Hydrolytic activity was assessed by visually comparing the control with active PARP2 (formation of a smear in SDS-PAGE) and its removal to restore the appearance of non-modified PARP2. PARG, TARG1, ARH1 and ARH3 were added at the beginning of the reaction and the result analysed with SDS-PAGE (Suppl. Fig. 2C). ARH3 was also tested at higher concentration and in that case, PARP2 sample was first automodified for $2 \mathrm{~h}$ as described above and then ARH3 was added along with PARP2 inhibitor Olaparib (15 nM) and incubated for $4 \mathrm{~h}$ at $37^{\circ} \mathrm{C}$ (Suppl. Fig. 2D). For TARG1 assay all proteins were mixed and the reaction was allowed to proceed at $37^{\circ} \mathrm{C}$ for $2 \mathrm{~h}$. Then, $20 \mathrm{nM}$ olaparib was added and the reaction continued for $1 \mathrm{~h}$. The samples were separated by SDS-PAGE and transferred to a nitrocellulose membrane (BioRad). The membrane was stained using Ponceau solution. Membrane was blocked with 5\% skimmed milk in TBS $+0.1 \%$ Tween-20 for $1 \mathrm{~h}$ and then incubated with AF1521 macrodomain tagged with GST overnight at $4^{\circ} \mathrm{C}$. 1:4000 anti-GST-HRP (Invitrogen) was used for detection and the reaction was developed using ECL (advansta) before imaging (Suppl. Fig. 2E). 


\section{Activity assay development}

$\alpha-\mathrm{NAD}^{+}$hydrolysis reactions were initially performed using black polypropylene U-shaped 96-well plates (Greiner, BioOne). The total volume of the reaction was $50 \mu \mathrm{L}$ and the reaction consisted of 100 $\mathrm{nM}$ macrodomain and $500 \mathrm{nM} \alpha-\mathrm{NAD}^{+}$in a buffer containing $25 \mathrm{mM}$ HEPES pH 7.5, $100 \mathrm{mM} \mathrm{NaCl}$ and $1 \mathrm{mg} / \mathrm{mL}$ bovine serum albumin (BSA). The plate was sealed and incubated at $25^{\circ} \mathrm{C}$ with constant shaking at $300 \mathrm{rpm}$ on a Biosan PST-60 HL plus thermo shaker (Biosan, LV). After the incubation, the remaining $\alpha-\mathrm{NAD}^{+}$was chemically modified to a fluorophore by adding $20 \mu \mathrm{L}$ of $20 \%(\mathrm{v} / \mathrm{v})$ acetophenone in absolute ethanol and $20 \mu \mathrm{l}$ of $2 \mathrm{M} \mathrm{KOH}$ followed by incubation at room temperature for $10 \mathrm{~min}$. Next, $90 \mu \mathrm{L}$ of $100 \%$ formic acid was added and the plate was incubated again at room temperature for $20 \mathrm{~min}$. The fluorescent signal was measured with Tecan Infinite M1000 pro plate reader using excitation at $372 \mathrm{~nm}$ and emission at $444 \mathrm{~nm}$. The fluorescence spectra is very similar to that reported for the fluorophore generated from similarly $\beta-N A D^{+}$(Supplementary Fig. 3). The background (signal from the buffer only) was subtracted and $\alpha-\mathrm{NAD}^{+}$hydrolysis activity was calculated by setting the conversion of the positive control with maximal signal to $0 \%$ (signal from the wells containing $\alpha-\mathrm{NAD}^{+}$only). In order to screen larger libraries and to reduce the consumable costs we transferred the assay into black polypropylene flat bottom 384-well plates (Greiner, BioOne). The assay conditions were kept same, but we reduced the volume to $10 \mu \mathrm{L}$ and used a Mantis microfluidic liquid handler (Formulatrix) to dispense the reagents to the plate. $\alpha-\mathrm{NAD}^{+}$was converted to a fluorophore by adding $4 \mu \mathrm{L}$ of $20 \%(\mathrm{v} / \mathrm{v})$ acetophenone and $4 \mu \mathrm{L}$ of $2 \mathrm{M} \mathrm{KOH}$ and finally adding 18 $\mu \mathrm{L}$ of $100 \%$ formic acid.

\section{Assay optimization}


The assay was optimized for MacroD2 and the optimization parameters included incubation time, concentrations of BSA and $\alpha-\mathrm{NAD}^{+}$. We first optimized the incubation time for the hydrolysis of $\alpha$ $\mathrm{NAD}^{+}$by testing different times from 3 min to $6 \mathrm{~h}$. Next, we optimized the BSA concentration in the assay buffer. The following BSA concentrations were used in the test: $1,0.9,0.75,0.6,0.4,0.25$ and $0.1 \mathrm{mg} / \mathrm{mL} . \alpha-\mathrm{NAD}^{+}$concentration was optimized by performing the hydrolysis using $0.5,1,2,4,6,8$ and $10 \mu \mathrm{M} \alpha-\mathrm{NAD}^{+}$. Finally, dimethyl sulfoxide (DMSO) tolerance of the assay was tested using 5, 3, 2, 1, 0.8, 0.4 and $0.1 \%$ DMSO concentrations.

The assay was validated on 96-well plates by measuring the repeatability of the maximal and minimal signals between different wells, plates, and days. In total, five plates were prepared, each of them containing 40 maximal and minimal signal points and 10 blank wells. For 384 well plates, five control plates were prepared containing 176 maximal and minimal signal points and 32 blank wells. These data points were used separately for both maximal and minimal signals to calculate well-to-well, plate-toplate and day-to-day mean signal, standard deviation and coefficient of variations (CVs). Three of the plates were measured on the first day, one plate was measured on the second day and the final plate was measured on the third day. The overall quality of the assay was determined with common statistical parameters: signal-to-background (S/B), signal-to-noise $(\mathrm{S} / \mathrm{N})$ and screening window coefficient $\left(Z^{\prime}\right)^{23,24}$.

\section{Screening of compounds}


1108 compounds of the Tocriscreen Mini (Tocris Bioscience) were screened against MacroD2. Each compound was screened in duplicates at a single concentration of $10 \mu \mathrm{M}$. The compounds $(0.01 \mu \mathrm{L})$ were supplied on the 384-well plates (Greiner, BioOne) by the Institute for Molecular Medicine, Finland (FIMM) using the Echo acoustic liquid dispenser (Labcyte, Sunnyvale, CA). $4 \mu \mathrm{L}$ of MacroD2 (final concentration $0.1 \mu \mathrm{M}$ ) and $5 \mu \mathrm{L}$ of $\alpha-\mathrm{NAD}^{+}$(final concentration $0.5 \mu \mathrm{M}$ ) added to each plate in assay buffer (25 mM HEPES pH 7.5, $100 \mathrm{mM} \mathrm{NaCl}$, and $1 \mathrm{mg} / \mathrm{mL} \mathrm{BSA}$ ) and incubated for $3 \mathrm{~h}$ at room temperature followed by an addition of $4 \mu \mathrm{L}$ of $20 \%(\mathrm{v} / \mathrm{v})$ acetophenone in absolute ethanol and $4 \mu \mathrm{L} 2$ $\mathrm{M} \mathrm{KOH}$. After that, the plate was incubated at room temperature for 10 minutes followed by an addition of $18 \mu \mathrm{L}$ of $100 \%$ formic acid. Finally, the plate was incubated at room temperature for 20 min and measured with Tecan Infinite M1000 pro plate reader. Each plate contained blank wells (assay buffer only), positive control (assay buffer with $\alpha-\mathrm{NAD}^{+}$) and negative control (assay buffer with $\alpha$ $\mathrm{NAD}^{+}$and MacroD2). The positive and negative controls were used to calculate the overall conversion. Compounds $60 \%$ inhibition, corresponding to $3 \sigma$ from the mean, to the activity of MacroD2 were considered as hit compounds. In order to assess the precision of the assay, a Pearson correlation was determined by plotting the inhibition- $\%$ produced by each of the two compound replicates. The hit compounds obtained from the primary screening were re-screened in quadruplicate at concentrations of 1 and $10 \mu \mathrm{M}$. In order to detect possible fluorescence signal produced by a hit compound, compound controls (no MacroD2 present) were included to this secondary screening.

\section{Potency measurements}


The dose-response curves were measured three times in quadruplicate from $100 \mu \mathrm{M}$ to $0.003 \mu \mathrm{M}$ using half-log dilutions. The compounds and macrodomains were diluted with the assay buffer $(25 \mathrm{mM}$ HEPES pH 7.5, $100 \mathrm{mM} \mathrm{NaCl}, 1 \mathrm{mg} / \mathrm{mL}$ BSA). $1 \mu \mathrm{L}$ of compound was manually transferred to the assay plate followed by addition of $4 \mu \mathrm{L}$ of macrodomain (final concentration $0.1 \mu \mathrm{M}$ ) and $5 \mu \mathrm{L}$ of $\alpha$ $\mathrm{NAD}^{+}$(final concentration $0.5 \mu \mathrm{M}$ ) using the Mantis microfluidic liquid handler (Formulatrix). The plate was incubated for $1 \mathrm{~h}$ at room temperature before the chemical reaction was performed. The doseresponse curves were fitted using a non-linear regression analysis with GraphPad prism version 5.04 for windows (GraphPad Software, Inc., USA).

\section{AlphaScreen assay}

An AlphaScreen assay for MAR hydrolyzing macrodomains was used as an orthogonal assay to determine the $\mathrm{IC}_{50}$ values of the hit compounds ${ }^{21}$. The assay is measuring hydrolysis of Serine/threonine-protein kinase 2 (SRPK2) kinase linked biotinylated ADPr. The compounds and proteins were diluted with the assay buffer (25 mM HEPES, $\mathrm{pH} 7.5,100 \mathrm{mM} \mathrm{NaCl}$ and $1 \mathrm{mg} / \mathrm{mL}$ BSA). The compounds were measured in quadruplicates with half-logarithmic dilutions from $320 \mu \mathrm{M}$ to $3.2 \mathrm{nM}$ and were transferred to the 384 -well AlphaScreen plate (PerkinElmer). $7.5 \mu \mathrm{L}$ of MacroD1 (final concentration $800 \mathrm{nM}$ ) or MacroD2 (final concentration $400 \mathrm{nM}$ ) and $7.5 \mu \mathrm{L}$ of biotin monoADP-ribosyl-SRPK2 (final concentration $50 \mathrm{nM}$ ) were added to the assay plate and incubated for 80 min at room temperature. After that $10 \mu \mathrm{L}$ of nickel chelate acceptor and streptavidin donor bead mixture (final concentration $5 \mu \mathrm{g} / \mathrm{mL}$ ) were added to the assay plate. The plate was incubated at room temperature for $3 \mathrm{~h}$ in dark. Luminescence was read with a Tecan Infinite M1000 Pro plate reader using the AlphaScreen detection module. The dose-response curves were fitted using a four-parameter nonlinear regression analysis with GraphPad prism version 5.04 for windows (GraphPad Software, Inc., USA). 


\section{Differential scanning fluorimetry}

To study direct binding of the hit molecules to MacroD1 and MacroD2 we used differential scanning fluorimetry (DSF). All the samples were prepared with the buffer (25 mM HEPES pH 7.5, $150 \mathrm{mM}$ $\mathrm{NaCl}$ ) and the sample volumes were $30 \mu \mathrm{L}$ containing $0.3 \mathrm{mg} / \mathrm{mL}$ macrodomain and $100 \mu \mathrm{M}$ compound and 5x SYPRO Orange dye (ThermoFisher Scientific, USA). The melting curves for macrodomains were measured using Applied Biosystems 7500 Real Time PCR system (ThermoFisher

Scientific, USA) with the temperature range from $20{ }^{\circ} \mathrm{C}$ to $90{ }^{\circ} \mathrm{C}$ with $1 \mathrm{C}$ increment per minute. The GraphPad prism software (version 5.04 for windows, GraphPad Software, Inc., USA) was used to fit the melting curves.

\section{Isothermal titration calorimetry}

In order to measure affinities with isothermal titration calorimetry (ITC), MacroD1 and MacroD2 were first dialyzed in buffer (50 mM HEPES pH 7.5, $250 \mathrm{mM} \mathrm{NaCl}, 0.5 \mathrm{mM}$ TCEP) at $+4{ }^{\circ} \mathrm{C}$ with constant stirring overnight. $40 \mu \mathrm{M}$ protein and $500 \mu \mathrm{M}$ of compounds were prepared in dialysis buffer for each reaction. For later experiments compound concentration was reduced to $100 \mu \mathrm{M}$ to eliminate compound precipitation. Binding experiments were carried out on a MicroCal iTC 200 (GE Healthcare) instrument. All titration experiments were performed in dialysis buffer at $25{ }^{\circ} \mathrm{C}, 11.4 \mu \mathrm{Cal} / \mathrm{s}$ reference power and $500 \mathrm{rpm}$ stirring speed. Each titration volume was $2.4 \mu \mathrm{L}$ followed by 17 identical cycles with a duration of $4.8 \mathrm{~s}$ between each titration and $180 \mathrm{~s}$ between injections to allow the baseline to stabilize.

\section{Mass spectrometry}


For the mass spectrometric characterization, the hit compounds were diluted from $10 \mathrm{mM}$ stocks in $100 \%$ DMSO to the concentrations of $10 \mu \mathrm{M}$ and $100 \mu \mathrm{M}$ with three solutions: $50 \%(\mathrm{v} / \mathrm{v})$ aqueous acetonitrile, $50 \%(\mathrm{v} / \mathrm{v})$ aqueous methanol and $10 \mathrm{mM}$ ammonium acetate in water. The samples were ionized in presence and absence of $1 \%(\mathrm{v} / \mathrm{v})$ formic acid with direct infusion using electrospray ionization (ESI) in positive mode. The ions were detected with Thermo Orbitrap Fusion Lumos Tribrid.

\section{Results}

\section{Optimization of the activity assay}

The assay conditions including time for incubation and concentrations of the reaction components affecting enzyme activity were optimized. It is important to note that $\alpha-\mathrm{NAD}^{+}$concentration significantly affected the substrate conversion percentage, which determines the signal window. At 0.5 $\mu \mathrm{M}$ concentration $70 \%$ conversion was achieved, while higher substrate concentration reduced conversion and limited usability of the assay (e.g. $20 \%$ at $10 \mu \mathrm{M}$ substrate; Fig. 1A). ADPr produced from $\alpha-\mathrm{NAD}^{+}$acts as an inhibitor for the macrodomain since the ADPr $\mathrm{K}_{\mathrm{D}}$ for MacroD1 and MacroD2 are reported to be 0.9 and $0.15 \mu \mathrm{M}$, respectively ${ }^{25}$. Hence, due to the product inhibition, it is essential for a robust screening assay to use a low substrate concentration. 
The enzyme activity was measured with $500 \mathrm{nM} \alpha-\mathrm{NAD}^{+}$in different time points with a range from 6$0.05 \mathrm{~h}$ and we selected $3 \mathrm{~h}$ incubation time for MacroD2 as it gave a 70\% substrate conversion (Fig. 1B), which will result in robust assay based on our previous experiments with PARP/ARTD enzymes ${ }^{26}$. BSA was included in the previous AlphaScreen activity assay we developed and we next optimized its concentration for the fluorescence assay ${ }^{21}$. Increased BSA concentration increased MacroD2 activity and BSA was shown to be essential for stable activity. $1 \mathrm{mg} / \mathrm{mL}$ concentration was selected to be used in further experiments (Fig. 1C). Small molecule compounds are typically dissolved in DMSO in the libraries and DMSO may affect the enzymatic activity. We tested concentrations up to 5\% DMSO and it did not significantly affect substrate conversion making the assay readily suitable for compound screening (Fig. 1D).

\section{Assay performance}

Assay quality was validated for screening by testing changes in plate-to-plate and day-to-day maximal and minimal signals over five different plates. The assay was first run and evaluated using manual pipetting on 96-well plates and it showed robust statistics with acceptable $\mathrm{S} / \mathrm{B}$ and $\mathrm{S} / \mathrm{N}$ values as well as $Z^{\prime}$ of 0.7 indicating a good screening assay (Table 1) ${ }^{23,24}$. Scaling of the assay down to 384-well plates and $10 \mu \mathrm{L}$ volume with the help of automated dispenser affected the values as $\mathrm{S} / \mathrm{N}$ increased from 16 to 27 while there was a small decrease in the average $Z^{\prime}$ value (0.6). Despite the above, the overall statistics indicate the assay to be suitable for the compound screening in both formats (Table 1).

\section{Validatory screening}


In order to test the assay capability in compound screening, we used the assay to screen the Tocriscreen Mini library in 384-well format. 23 compounds showed higher fluorescence compared to the control and after removing these false positives 20 compounds were identified as initial hits for MacroD2 using a 60\% hit limit (Fig. 2A). Overall, the assay performance was good in the screening with an average $\mathrm{Z}$ ' of 0.78 over controls on seven plates. The screening was carried out in duplicates located on different halves of the plate and the Pearson correlation coefficient $\left(\mathrm{r}^{2}\right)$ was 0.89 confirming a good accuracy of the assay, while a few outliers could be identified in one or the other set. Almost the same set of hit compounds would have become hits even if the duplicates were treated independently (Fig. 2B).

Next, we carried out a secondary screening for 20 hit compounds at $1 \mu \mathrm{M}$ and $10 \mu \mathrm{M}$ concentrations and found that 16 out of 20 compounds produced fluorescence with the used wavelengths. The inherent fluorescence mimics inhibition (resulting in $1.4 \%$ false hit rate) and can be controlled in the primary screen by using separate wells containing $\mathrm{NAD}^{+}$and compound but no enzyme. Four compounds inhibited the enzymatic activity of MacroD2 more than $60 \%$ at $10 \mu \mathrm{M}$ concentration and three of them inhibited the enzymatic activity more than $27 \%$ at $1 \mu \mathrm{M}$ concentration. These compounds were NSC 95397 (2,3-bis[(2-Hydroxyethyl)thiol]-1,4-naphthoquinone), BI-78D3 (4-(2,3-Dihydro-1, 4benzodioxin-6-yl)-2,4-dihydro-5-[(5-nitro-2-thiazolyl)thio]-3H-1,2,4-triazol-3-one) and BAY 11-7085 ((2E)-3-[[4-(1,1-Dimethylethyl)phenyl]sulfonyl]-2 propenenitrile).

\section{Concentration-response measurements}


Concentration response measurements could be carried out to two out of the three hit compounds as NSC 95397 precipitated in the assay buffer. No further experiments were carried out with the compound and we performed $\mathrm{IC}_{50}$ measurements for BI-78D3 and BAY 11-7085. Based on the average of three measurements, the $\mathrm{IC}_{50}$ values of $\mathrm{BI}-78 \mathrm{D} 3$ for MacroD1 and MacroD2 were $1.5 \mu \mathrm{M}$ and 3.2 $\mu \mathrm{M}$, respectively. BAY 11-7085 was slightly more potent having $0.9 \mu \mathrm{M}$ and $3.1 \mu \mathrm{M} \mathrm{IC}_{50}$ values for MacroD1 and MacroD2, respectively. In addition, we carried out the measurement also for ADPr as a control and the $\mathrm{IC}_{50}$ values of $\mathrm{ADPr}$ for MacroD1 and MacroD2 were $1.6 \mu \mathrm{M}$ and $1.4 \mu \mathrm{M}$, respectively (Fig. 3; Table 2). Results for all the individual potency measurements of the compounds with MacroD1 and MacroD2 are shown in the supplementary information (Suppl. Fig. 4 \& 5). We further validated the $\mathrm{IC}_{50}$ values by using our AlphaScreen assay for MAR hydrolyzing macrodomains and the $\mathrm{IC}_{50}$ values obtained from the orthogonal assay were similar for BI-78D3 having $2.0 \mu \mathrm{M}$ and $1.5 \mu \mathrm{M}$ $\mathrm{IC}_{50}$ values for MacroD1 and MacroD2, respectively (Table 2; Suppl. Fig. 6). The $\mathrm{IC}_{50}$ values of BAY 11-7085 were $5.6 \mu \mathrm{M}$ and $3.3 \mu \mathrm{M}$ for MacroD1 and MacroD2, respectively and the $\mathrm{IC}_{50}$ values of ADPr for MacroD1 and MacroD2 were $0.8 \mu \mathrm{M}$ and $1.2 \mu \mathrm{M}$ respectively.

\section{Orthogonal assays and compound characterization}


In a thermal shift assay we measured the melting curves for MacroD1 and MacroD2 in absence and presence of BI-78D3, BAY 11-7085 and ADPr (Suppl. Fig. 7). Based on the shifts of the $\mathrm{T}_{\mathrm{M}}$ values $\left(\Delta \mathrm{T}_{\mathrm{M}}\right)$ presented in (Table 2), BI-78D3 destabilized both MacroD1 and MacroD2. BAY 11-7085 slightly stabilized MacroD1 $\left(\Delta \mathrm{T}_{\mathrm{M}}=0.44^{\circ} \mathrm{C}\right)$, but clearly destabilized MacroD2. ADPr on the other hand showed a clear stabilization for both MacroD1 $\left(\Delta \mathrm{T}_{\mathrm{M}}=2.41{ }^{\circ} \mathrm{C}\right)$ and $\operatorname{MacroD} 2\left(\Delta \mathrm{T}_{\mathrm{M}}=3.93{ }^{\circ} \mathrm{C}\right)$. We further tested the binding of the compounds using ITC. Both compounds showed precipitation in high concentration $(500 \mu \mathrm{M})$ and therefore the experiments were also done in lower $100 \mu \mathrm{M}$ concentration. Despite the efforts we were unable to detect any binding of BI-78D3 and BAY 11-7085 to MacroD1 and MacroD2, while ADPr showed a binding curve similar to reported in the literature and $\mathrm{K}_{\mathrm{D}}$ values of 1.34 and $0.30 \mu \mathrm{M}$, respectively (Suppl. Fig. 8) ${ }^{25}$. Because we did not observe any binding of the hit compounds in ITC, we performed mass spectrometric characterizations for the compounds. Even though the compounds were measured with low and high concentrations $(10 \mu \mathrm{M}$ and $100 \mu \mathrm{M}$, respectively) and under various conditions, we failed to detect them with mass spectrometry (MS) indicating that they would not be stable and likely degraded. Based on that, it is also possible that some unidentified degradation products caused the destabilization of MacroD2.

\section{Assay for other ADP-ribosylhydrolases}

While we focused our efforts to MacroD2 in the validatory screening, we also tested the applicability of the $\alpha-\mathrm{NAD}^{+}$hydrolysis reaction with range of human enzymes reported as ADP-ribosyl hydrolases. In addition to MacroD1 and MacroD2, known macrodomain containing ADP-ribosylhydrolases TARG1 and PARG were active in the assay (Fig. 4). The activity is lower than for MacroD1 and MacroD2 indicating that assay conditions used would not be optimal for these enzymes and would require further optimization. ARH family enzymes (ARH1, ARH2 and ARH3) were also tested for $\alpha$ $\mathrm{NAD}^{+}$hydrolysis. Consistent with the literature only ARH3 showed robust activity in the assay at the same level as MacroD1 and MacroD2 (Fig. 4) ${ }^{19}$. 


\section{Discussion}

Fluorescence-based activity assays using $\beta-\mathrm{NAD}^{+}$were previously developed aiming for mono or polyADP-ribosyltransferases ${ }^{26-28}$. During the ADP-ribosyltransferase reaction, a proximal ribose is attached to the protein side chain from the opposite side of the nicotinamide attached to the ribose 1 '- position. Macrodomains are designed to bind protein linked ADPr groups and therefore they are capable of binding $\alpha-\mathrm{NAD}^{+}$, which mimics protein linked ADPr. Some macrodomains can hydrolyze $\alpha-\mathrm{NAD}^{+}$and this opened up the way to develop an assay for hydrolyzing macrodomains by chemically converting $\alpha$ $\mathrm{NAD}^{+}$to a fluorophore with similar fluorescence spectra as reported for $\beta-\mathrm{NAD}^{+27}$.

Some assays have been developed in the past to screen inhibitors and monitor activity and functions of different macrodomains. For example, AlphaScreen based inhibitor screening strategy and strategy related to the identification of enzymatic activity were developed for PARP14-macrodomains that act as readers of ADP-ribosylation ${ }^{29,30}$. Contrary to these, an AlphaScreen assay was established that specifically measures the hydrolyzing activity of MAR macrodomains onto protein-linked ADPr ${ }^{21}$. All the methods including AlphaScreen technology are bead-based that generate amplified signal in the presence of a specific biological interaction. Also immunoblotting, mass spectrometry and cell based assays have been used to study hydrolyzing and deacetylase activity of various macrodomains and ARHs ${ }^{31-33}$. In comparison to the previously used methods, the assay described here requires only addition and incubation steps with common reagents that together with high density microtiter plate compatibility result in assay costs of only $0.03 \mathrm{USD} /$ well excluding compound and dispensing costs. 
ADP-ribosylhydrolases are potential therapeutic targets and specific inhibitors would help to validate them. We described a simple, cost effective and easy to execute assay using active enzyme fragments that can be produced in E.coli. The assay was optimized for human MacroD2 and to validate the assay we screened a random compound library. This allowed us to evaluate potential limitations of the assay in screening campaigns that one should take into account. Notably, some small molecules showed fluorescence and interfered with the assay signal at the wavelengths used. On the other hand some compounds might have converted to a fluorophore during the chemical reaction. It is possible to assess these effects during hit validation step or by including a compound-only-controls in the primary screening. It should be noted also that one cannot overcome this limitation by increasing substrate concentration and subsequently the signal due to the product inhibition by ADPr. Despite this drawback the homogenous assay is inexpensive, easy to perform, and automate.

The validatory screening yielded three hits inhibiting MacroD2, and two were further validated in doseresponse measurements and in orthogonal assays. The identified micromolar inhibitors BI-78D3 and BAY 11-7085 are biologically active compounds belonging to different chemical classes. The compounds have a range of activities, but mainly identified as phosphatase and kinase inhibitors. BI$78 \mathrm{D} 3$ is reported as a competitive c-Jun N-terminal kinase (JNK) inhibitor and BAY 11-7085 inhibits TNF- $\alpha$-induced $1 \mathrm{kB} \alpha$ phosphorylation ${ }^{34-36}$. The compounds indeed displayed some inhibition towards both MacroD1 and MacroD2, but failed in orthogonal assays and were identified as protein destabilizers, which likely results in the inhibition seen in the primary assay. The negative orthogonal assays and quality control data showing likely compound degradation did not justify further studies with the hit compounds. However, identification of the hit compounds demonstrated the capability of the assay to identify inhibitors. Our results also show that the assay principle is applicable for all ADPr hydrolases accepting $\alpha-\mathrm{NAD}^{+}$as a substrate. The assay can therefore be readily established in laboratories to facilitate the development of chemical probes against these enzymes. 


\section{Acknowledgements}

We thank the FIMM (UH) Technology Centre and Dr. Laura Turunen for help in preparing the assay plates. We thank Dr. Teemu Haikarainen for help in cloning of PARG and Dr. Yashwanth Ashok for help in cloning of ARHs and for the production of Af1521. Contstruct encoding for His-GST-Af1521 was a generous gift from Dr. Arto Pulliainen. We also thank Dr. Ulrich Bergmann from Proteomics core facility for help in the Mass Spectrometry analysis of the inhibitors. The use of the facilities of the Biocenter Oulu Protein Crystallography core facility member of Biocenter Finland and Instruct-FI, is gratefully acknowledged. This work was funded by Academy of Finland (grant no. 287063 and 294085) and by Sigrid Jusélius foundation. 


\section{References}

1. Talhaoui, I.; Lebedeva, N. A.; Zarkovic, G.; et al. Poly(ADP-Ribose) Polymerases Covalently Modify Strand Break Termini in DNA Fragments in Vitro. Nucleic Acids Res. 2016, 44, 9279-9295.

2. Gupte, R.; Liu, Z.; Kraus, W. L. PARPs and ADP-Ribosylation: Recent Advances Linking Molecular Functions to Biological Outcomes. Genes Dev. 2017, 31, 101-126.

3. Munnur, D.; Ahel, I. Reversible Mono-ADP-Ribosylation of DNA Breaks. FEBS J. 2017, 284, 4002-4016.

4. Munnur, D.; Bartlett, E.; Mikolčević, P.; et al. Reversible ADP-Ribosylation of RNA. Nucleic Acids Res. 2019, 47, 5658-5669.

5. Palazzo, L.; Thomas, B.; Jemth, A.-S.; et al. Processing of Protein ADP-Ribosylation by Nudix Hydrolases. Biochem. J. 2015, 468, 293-301.

6. Rack, J. G. M.; Perina, D.; Ahel, I. Macrodomains: Structure, Function, Evolution, and Catalytic Activities. Annu. Rev. Biochem. 2016, 85, 431-454.

7. Feijs, K. L.; Forst, A. H.; Verheugd, P.; et al. Macrodomain-Containing Proteins: Regulating New Intracellular Functions of Mono (ADP-Ribosyl) Ation. Nat. Rev. Mol. Cell Biol. 2013, 14, 443.

8. Chen, D.; Vollmar, M.; Rossi, M. N.; et al. Identification of Macrodomain Proteins as Novel O-AcetylADP-Ribose Deacetylases. J. Biol. Chem. 2011, 286, 13261-13271.

9. Mo, Z.; Hu, M.; Yu, F.; et al. Leukemia-Related Protein 16 (LRP16) Promotes Tumor Growth and Metastasis in Pancreatic Cancer. OncoTargets Ther. 2018, Volume 11, 1215-1222.

10. Mohseni, M.; Cidado, J.; Croessmann, S.; et al. MACROD2 Overexpression Mediates Estrogen Independent Growth and Tamoxifen Resistance in Breast Cancers. Proc. Natl. Acad. Sci. 2014, 111, 17606-17611.

11. Sakthianandeswaren, A.; Parsons, M. J.; Mouradov, D.; et al. MACROD2 Deletions Cause Impaired PARP1 Activity and Chromosome Instability in Colorectal Cancer. Oncotarget 2018, 9.

12. Voorneveld, J.; Rack, J. G. M.; Ahel, I.; et al. Synthetic $\alpha$ - and $\beta$-Ser-ADP-Ribosylated Peptides Reveal $\alpha$ Ser-ADPr as the Native Epimer. Org. Lett. 2018, 20, 4140-4143.

13. Ono, T.; Kasamatsu, A.; Oka, S.; et al. The 39-KDa Poly(ADP-Ribose) Glycohydrolase ARH3 Hydrolyzes O-Acetyl-ADP-Ribose, a Product of the Sir2 Family of Acetyl-Histone Deacetylases. Proc. Natl. Acad. Sci. 2006, 103, 16687-16691. 
14. Kasamatsu, A.; Nakao, M.; Smith, B. C.; et al. Hydrolysis of $O$-Acetyl-ADP-Ribose Isomers by ADPRibosylhydrolase 3. J. Biol. Chem. 2011, 286, 21110-21117.

15. Mashimo, M.; Kato, J.; Moss, J. ADP-Ribosyl-Acceptor Hydrolase 3 Regulates Poly (ADP-Ribose) Degradation and Cell Death during Oxidative Stress. Proc. Natl. Acad. Sci. 2013, 110, 18964-18969.

16. Mashimo, M.; Kato, J.; Moss, J. Structure and Function of the ARH Family of ADP-Ribosyl-Acceptor Hydrolases. DNA Repair 2014, 23, 88-94.

17. Houl, J. H.; Ye, Z.; Brosey, C. A.; et al. Selective Small Molecule PARG Inhibitor Causes Replication Fork Stalling and Cancer Cell Death. Nat. Commun. 2019, 10, 5654.

18. Li, X.; Wu, Z.; An, X.; et al. Blockade of the LRP16-PKR-NF-KB Signaling Axis Sensitizes Colorectal Carcinoma Cells to DNA-Damaging Cytotoxic Therapy. eLife 2017, 6, e27301.

19. Stevens, L. A.; Kato, J.; Kasamatsu, A.; et al. The ARH and Macrodomain Families of $\alpha$-ADP-RiboseAcceptor Hydrolases Catalyze $\alpha-N A D+$ Hydrolysis. ACS Chem. Biol. 2019.

20. Obaji, E.; Haikarainen, T.; Lehtiö, L. Characterization of the DNA Dependent Activation of Human ARTD2/PARP2. Sci. Rep. 2016, 6, 34487.

21. Haikarainen, T.; Maksimainen, M. M.; Obaji, E.; et al. Development of an Inhibitor Screening Assay for Mono-ADP-Ribosyl Hydrolyzing Macrodomains Using Alphascreen Technology. SLAS Discov. Adv. Life Sci. RD 2018, 23, 255-263.

22. Liszczak, G.; Diehl, K. L.; Dann, G. P.; et al. Acetylation Blocks DNA Damage-Induced Chromatin ADPRibosylation. Nat. Chem. Biol. 2018, 14, 837-840.

23. Zhang, J.-H.; Chung, T. D.; Oldenburg, K. R. A Simple Statistical Parameter for Use in Evaluation and Validation of High Throughput Screening Assays. J. Biomol. Screen. 1999, 4, 67-73.

24. Inglese, J.; Johnson, R. L.; Simeonov, A.; et al. High-Throughput Screening Assays for the Identification of Chemical Probes. Nat. Chem. Biol. 2007, 3, 466.

25. Neuvonen, M.; Ahola, T. Differential Activities of Cellular and Viral Macro Domain Proteins in Binding of ADP-Ribose Metabolites. J. Mol. Biol. 2009, 385, 212-225.

26. Venkannagari, H.; Fallarero, A.; Feijs, K. L.; et al. Activity-Based Assay for Human Mono-ADPRibosyltransferases Aimed at Screening and Profiling Inhibitors. Eur. J. Pharm. Sci. 2013, 49, 148-156. 
27. Putt, K. S.; Hergenrother, P. J. An Enzymatic Assay for Poly (ADP-Ribose) Polymerase-1 (PARP-1) via the Chemical Quantitation of NAD+: Application to the High-Throughput Screening of Small Molecules as Potential Inhibitors. Anal. Biochem. 2004, 326, 78-86.

28. Narwal, M.; Fallarero, A.; Vuorela, P.; et al. Homogeneous Screening Assay for Human Tankyrase. $J$. Biomol. Screen. 2012, 17, 593-604.

29. Schuller, M.; Riedel, K.; Gibbs-Seymour, I.; et al. Discovery of a Selective Allosteric Inhibitor Targeting Macrodomain 2 of Polyadenosine-Diphosphate-Ribose Polymerase 14. ACS Chem. Biol. 2017, 12, 28662874.

30. Ekblad, T.; Verheugd, P.; Lindgren, A. E.; et al. Identification of Poly(ADP-Ribose) Polymerase Macrodomain Inhibitors Using an AlphaScreen Protocol. SLAS Discov. Adv. Life Sci. RD 2018, 23, 353362.

31. Hirsch, B. M.; Burgos, E. S.; Schramm, V. L. Transition-State Analysis of 2- $O$-Acetyl-ADP-Ribose Hydrolysis by Human Macrodomain 1. ACS Chem. Biol. 2014, 9, 2255-2262.

32. Haikarainen, T.; Lehtiö, L. Proximal ADP-Ribose Hydrolysis in Trypanosomatids Is Catalyzed by a Macrodomain. Sci. Rep. 2016, 6, 24213.

33. Drown, B. S.; Shirai, T.; Rack, J. G. M.; et al. Monitoring Poly(ADP-Ribosyl)Glycohydrolase Activity with a Continuous Fluorescent Substrate. Cell Chem. Biol. 2018, 25, 1562-1570.e19.

34. Peyregne, V. P.; Kar, S.; Ham, S. W.; et al. Novel Hydroxyl Naphthoquinones with Potent Cdc25 Antagonizing and Growth Inhibitory Properties. Mol. Cancer Ther. 2005, 4, 595-602.

35. Kwon, O.-J.; Valdez, J. M.; Zhang, L.; et al. Increased Notch Signalling Inhibits Anoikis and Stimulates Proliferation of Prostate Luminal Epithelial Cells. Nat. Commun. 2014, 5, 4416.

36. Lahiri, S. K.; Lu, H.; Mukherjee, D.; et al. ERK2 Phosphorylates Krüppel-like Factor 8 Protein at Serine 48 to Maintain Its Stability. Am. J. Cancer Res. 2016, 6, 910. 
Table 1. Assay Performance

\begin{tabular}{|l|c|c|}
\hline Statistical parameters & 96-well plate & 384-well plate \\
\hline S/B & $2.95 \pm 0.15$ & $3.83 \pm 0.71$ \\
\hline $\mathrm{S} / \mathrm{N}$ & $15.9 \pm 3.6$ & $26.9 \pm 4.7$ \\
\hline$Z^{\prime}$ & $0.7 \pm 0.1$ & $0.6 \pm 0.1$ \\
\hline Well-to-well CV (Max/min, \%) & $3.82 \pm 0.85 / 5.62 \pm 1.53$ & $6.98 \pm 1.62 / 10.48 \pm 1.08$ \\
\hline Plate-to-plate CV (\%)* & 3.6 & $7.8-11.7$ \\
\hline Day-to-day CV (\%)* & $5.3-8.9$ & \\
\hline
\end{tabular}

*Calculated from Z' values. 
Table 2. Thermal shifts and potency measurements for ADPr and hit compounds.

\begin{tabular}{|c|c|c|c|c|c|c|}
\hline \multirow[b]{2}{*}{ Compound } & \multicolumn{3}{|c|}{ MacroD1 } & \multicolumn{3}{|c|}{ MacroD2 } \\
\hline & $\begin{array}{l}\text { Thermal } \\
\text { shift } \Delta \mathrm{T}_{\mathrm{M}} \\
\left({ }^{\circ} \mathrm{C}\right)\end{array}$ & $\begin{array}{c}\mathrm{IC}_{50}(\mu \mathrm{M}) \\
\left(\mathrm{pIC}_{50} \pm \mathrm{SEM}\right)\end{array}$ & $\begin{array}{l}\text { AlphaScreen } \\
\qquad \mathrm{IC}_{50} \\
(\mu \mathrm{M})\end{array}$ & $\begin{array}{c}\text { Thermal } \\
\text { shift } \Delta \mathrm{T}_{\mathrm{M}} \\
\left({ }^{\circ} \mathrm{C}\right)\end{array}$ & $\begin{array}{c}\mathrm{IC}_{50}(\mu \mathrm{M}) \\
\left(\mathrm{pIC}_{50} \pm \mathrm{SEM}\right)\end{array}$ & $\begin{array}{l}\text { AlphaScreen } \\
\qquad \mathrm{IC}_{50} \\
(\mu \mathrm{M})\end{array}$ \\
\hline ADPr & $2.41 \pm 0.11$ & $\begin{array}{c}1.6 \\
(5.81 \pm 0.13)\end{array}$ & 0.8 & $3.93 \pm 0.07$ & $\begin{array}{c}1.4 \\
(5.86 \pm 0.11)\end{array}$ & 1.2 \\
\hline BI-78D3 & $-2.88 \pm 2.05$ & $\begin{array}{c}1.5 \\
(5.82 \pm 0.04)\end{array}$ & 2.0 & $-7.17 \pm 0.28$ & $\begin{array}{c}3.2 \\
(5.50 \pm 0.09)\end{array}$ & 1.5 \\
\hline $\begin{array}{c}\text { BAY 11- } \\
7085\end{array}$ & $0.44 \pm 0.30$ & $\begin{array}{c}0.9 \\
(6.06 \pm 0.07)\end{array}$ & 5.6 & $-8.71 \pm 0.69$ & $\begin{array}{c}3.1 \\
(5.52 \pm 0.14)\end{array}$ & 3.3 \\
\hline
\end{tabular}


A

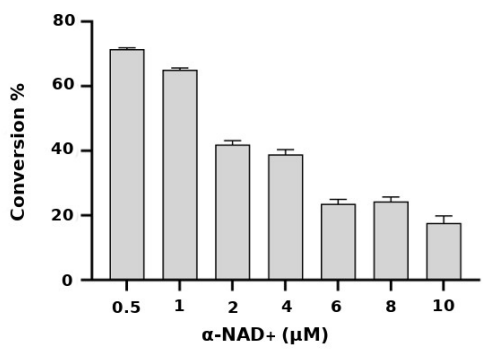

c

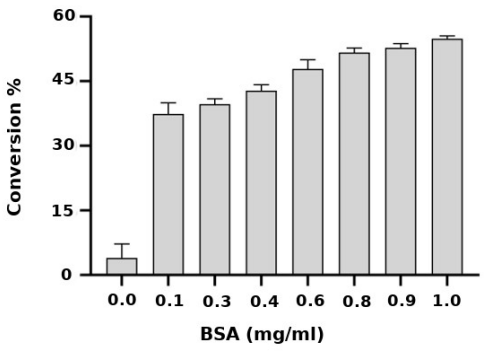

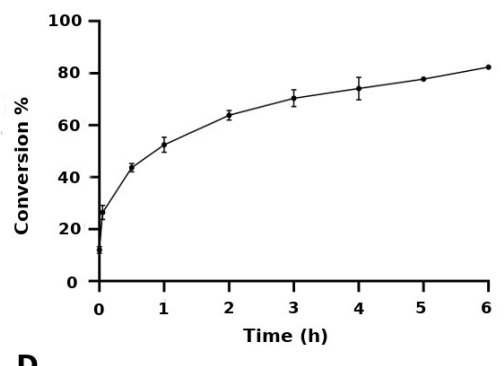

D

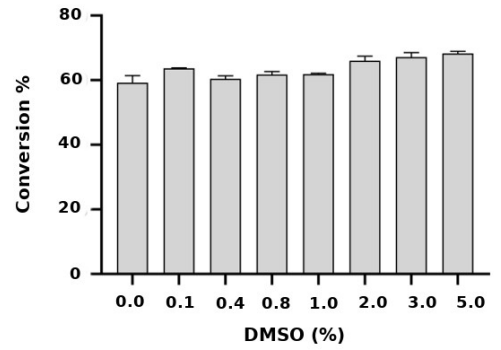

Figure 1. Optimization of the assay conditions for MacroD2. (A) $\alpha-\mathrm{NAD}^{+}$concentration dependent activity test. (B) Time dependent activity test. (C) BSA concentration effect. (D) DMSO tolerance of the assay. All measurements were carried out in quadruplicates and reported as mean $\pm \mathrm{SD}$. 
$\mathbf{A}$

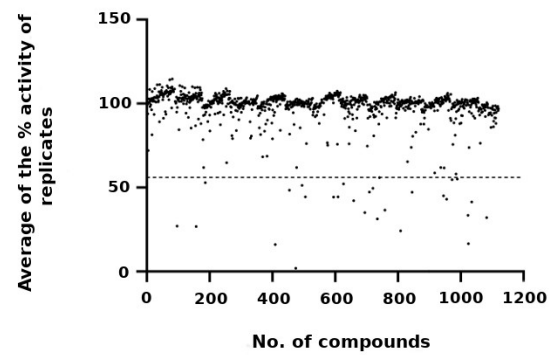

B

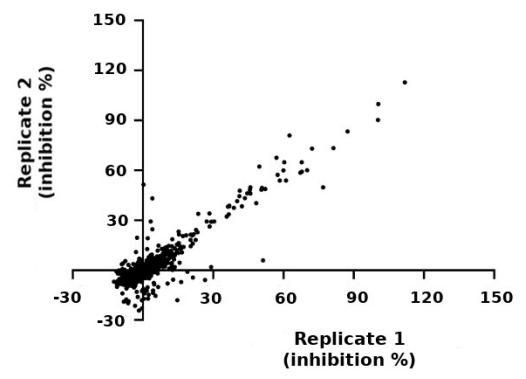

Figure 2. Screening of TocrisScreen Mini library with MacroD2 at $10 \mu \mathrm{M}$ concentration. (A) Mean relative activity of two data points is shown. (B) Pearson correlation of the replicates is shown for MacroD2 and the correlation coefficient $\left(\mathrm{r}^{2}\right)$ was 0.89 . 
A
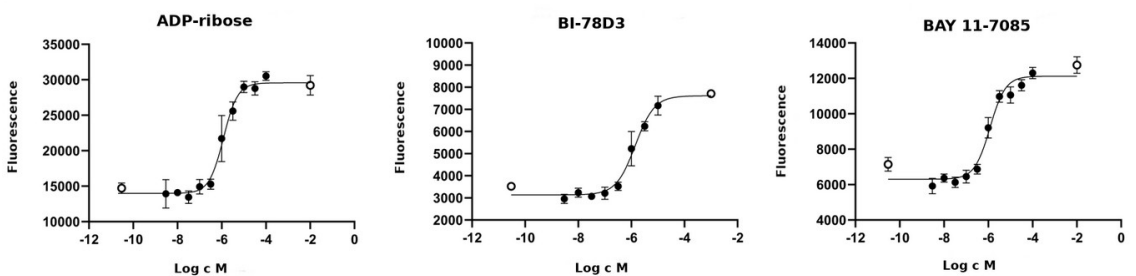

B
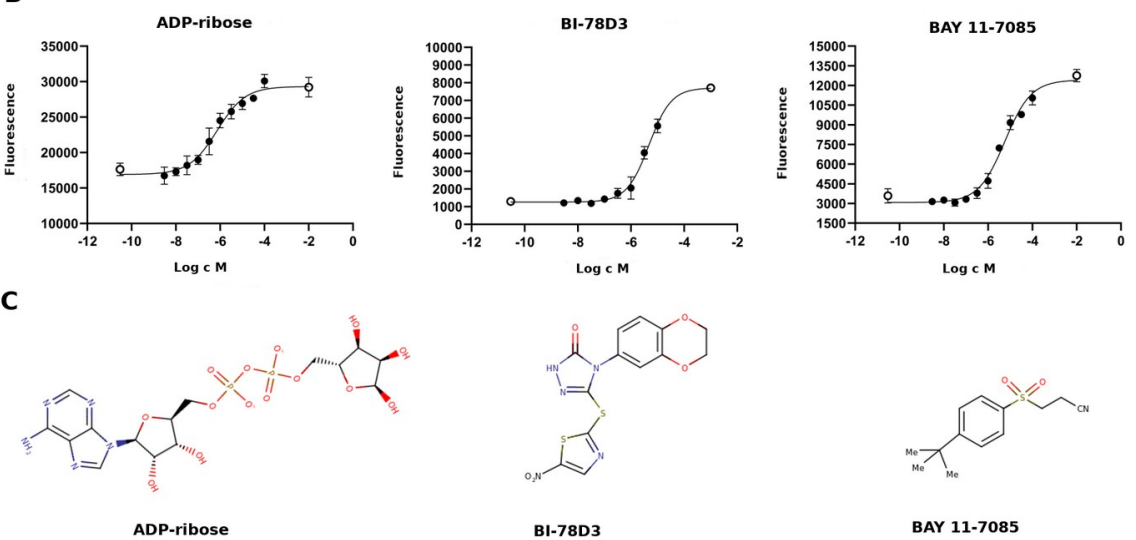

Figure 3. Measurements of half-maximal inhibitory concentrations of the inhibitors. Dose-response curves for the inhibitors (A) MacroD1 and (B) MacroD2. (C) Structures of the compounds. All measurements were carried out in quadruplicates using half-logarithimic dilutions starting from 100 $\mu \mathrm{M}$ to $0.003 \mu \mathrm{M}$ for each curve. Data reported as mean $\pm \mathrm{SD}$. 


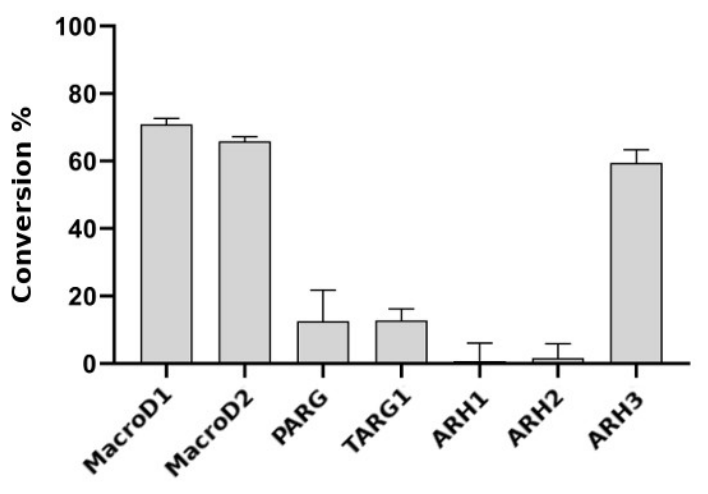

Figure 4. Activity test of human macrodomains and ADP-ribosylhydrolases utilizing the assay. $\alpha$ $\mathrm{NAD}^{+}$hydrolyzing activity was tested with selected enzymes at $0.5 \mu \mathrm{M}$ substrate concentration. Enzymes were tested at $100 \mathrm{nM}$ concentration. Data reported as mean values of 12-16 replicates $\pm \mathrm{SD}$. 


\section{Supplementary Material}

\section{Activity-based Screening Assay for Mono-ADP-ribosylhydrolases}

Sarah Wazir, Mirko M. Maksimainen, Heli I. Alanen, Albert Galera-Prat and Lari Lehtiö*

Faculty of Biochemistry and Molecular Medicine \& Biocenter Oulu, University of Oulu, Oulu, Finland

\section{Content:}

Figure S1. Fluorescence-based activity assay principle.

Figure S2. Quality of used recombinant proteins.

Figure S3. Fluorescence spectra for the fluorophores generated from $\alpha-\mathrm{NAD}^{+}$and $\beta-\mathrm{NAD}^{+}$.

Figure S4. $\mathrm{IC}_{50}$ measurements of MacroD1 for ADPr and hit compounds.

Figure S5. $\mathrm{IC}_{50}$ measurements of MacroD2 for ADPr and hit compounds.

Figure S6. $\mathrm{IC}_{50}$ measurement of ADPr and hit compounds utilizing AlphaScreen assay.

Figure S7. Thermal shift assay of MacroD1 and MacroD2 for ADPr and hit compounds.

Figure S8. Isothermal titration calorimetry profile of ADPr with MacroD1 and MacroD2. 
A

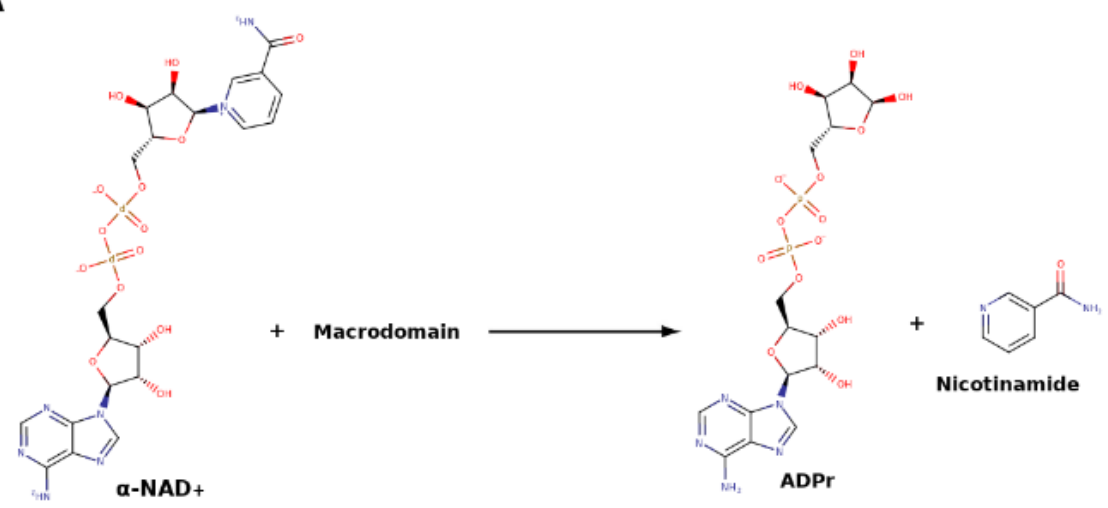

$\mathbf{B}$

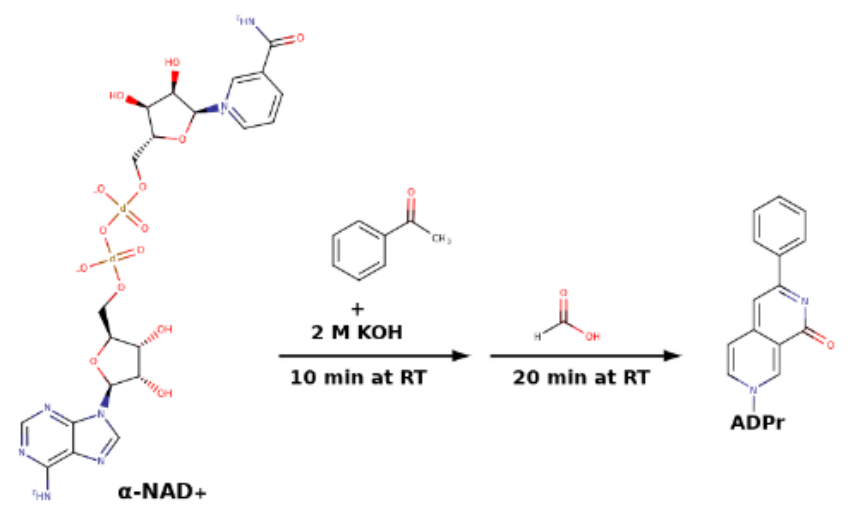

Supplementary figure 1. Fluorescence-based activity assay principle for MAR hydrolyzing macrodomains. (A) Enzymatic reaction carried out by MAR hydrolyzing macrodomains. (B) Chemical conversion of $\alpha-\mathrm{NAD}^{+}$ to a fluorescent analog. 
A

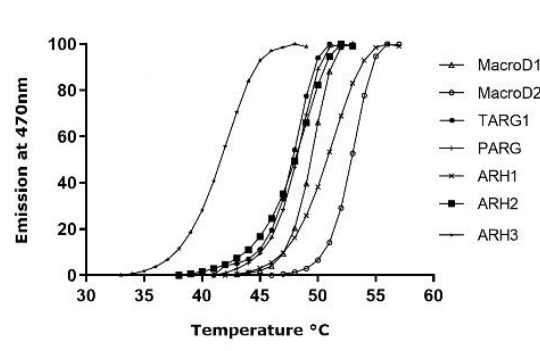

B

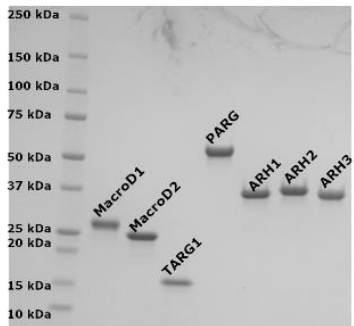

c

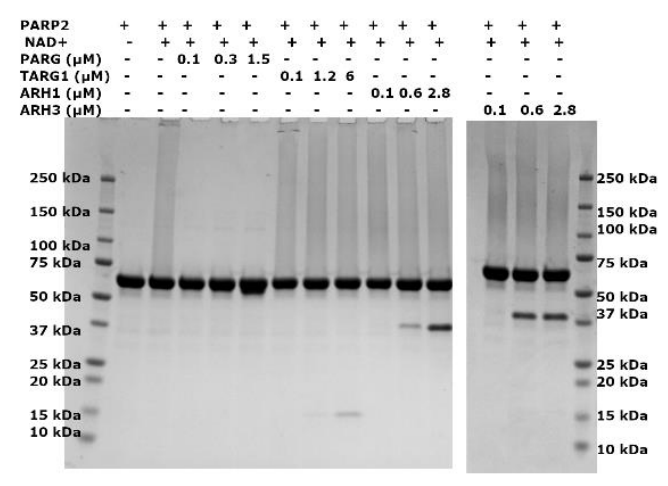

D

E
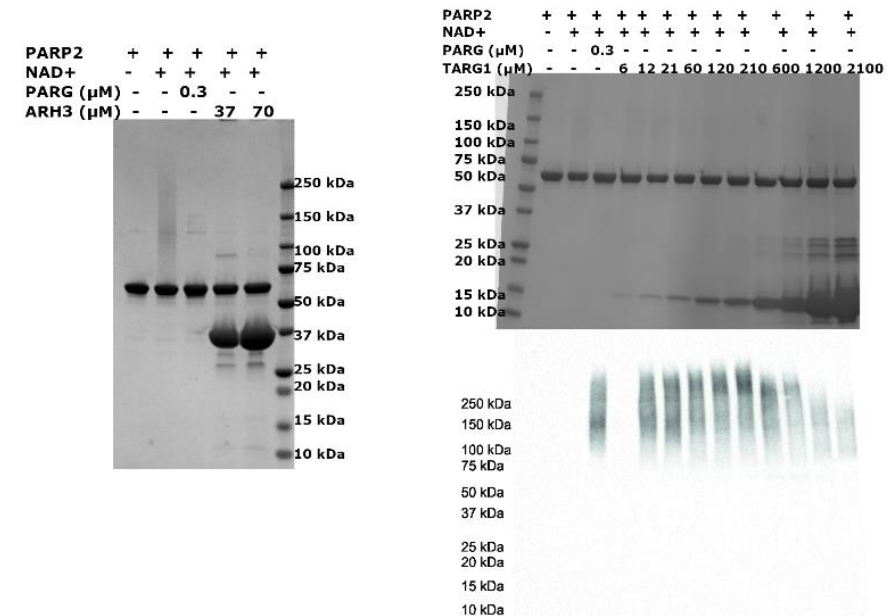

Supplementary figure 2. (A) Thermal shift assay for macrodomains. Samples were prepared in triplicates. Data points are shown on each curve and means for all replicates of control and for the macrodomains are fitted to obtain the curves. (B) SDS-PAGE gel showing final purification of all the seven macrodomains utilized in the assay. (C) SDS-PAGE of PARG, TARG1, ARH1 and ARH3 hydrolytic activity on PAR from auto-modified PARP2. PARG (lanes 3-5) effectively hydrolyses PAR, while TARG1 (lanes 6-8), ARH1 (lanes 9-11) and ARH3 (12-14) had no visible effect under these conditions. (D) SDS-PAGE of ARH3 acting on PAR from auto-modified PARP2. ARH3 (lanes 4 and 5) can remove PAR from auto-modified PARP2. (E) Ponceau stained membrane (top) and WB using AF1521 as a probe (bottom) of TARG1 with auto-modified PARP2. High concentration of TARG1 (last lanes) can reduce PARP2 smear indicating that the protein is active. 
A

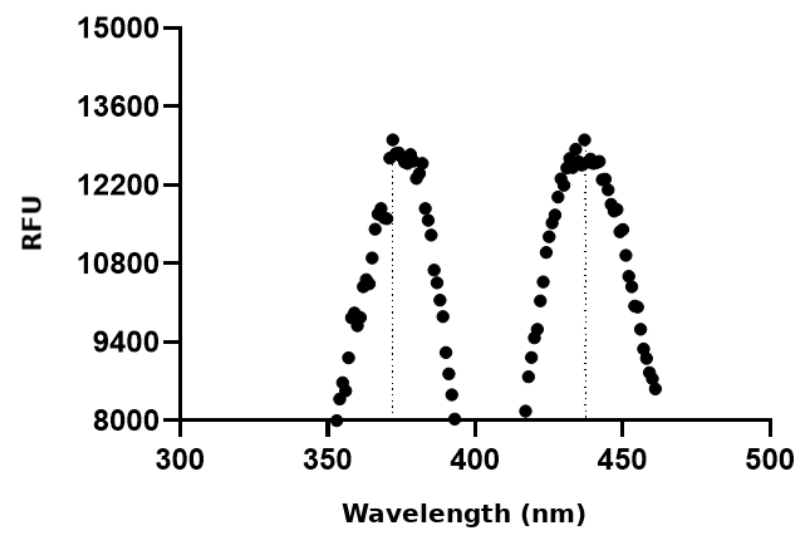

B

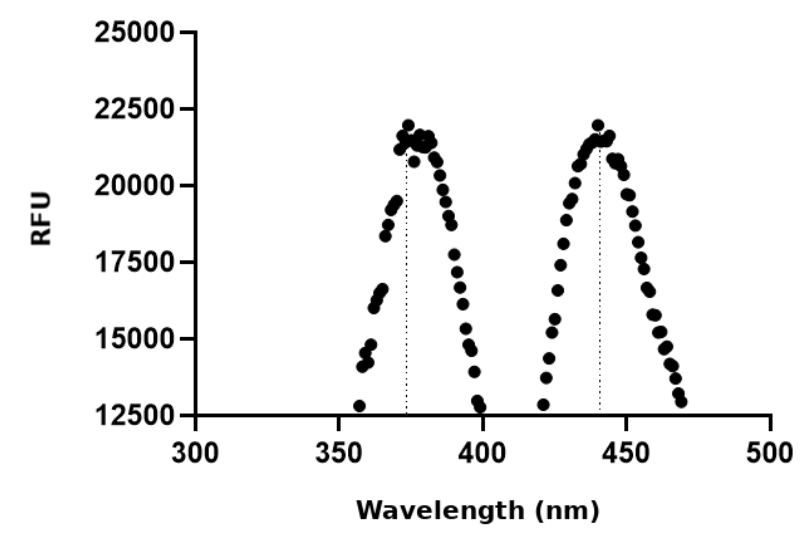

Supplementary figure 3: Fluorescence spectra for (A) fluorophore generated from $\alpha-\mathrm{NAD}^{+}$and (B) fluorophore generated $\beta-\mathrm{NAD}^{+}$. Wavelengths used on the assay, excitation at $372 \mathrm{~nm}$ and emission at $444 \mathrm{~nm}$, are marked to the figures with vertical dashed lines. 


\section{MacroD1}

A
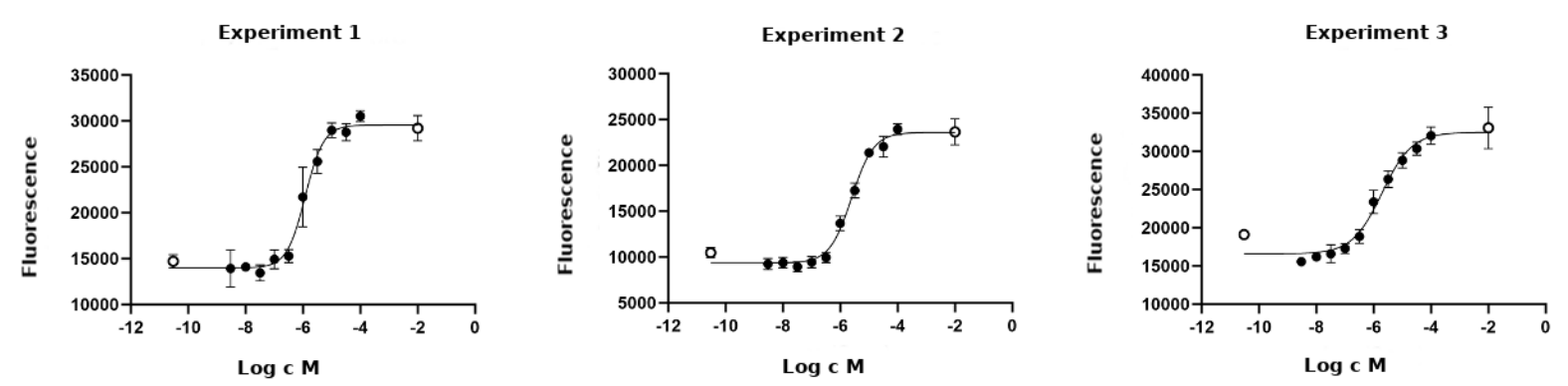

B
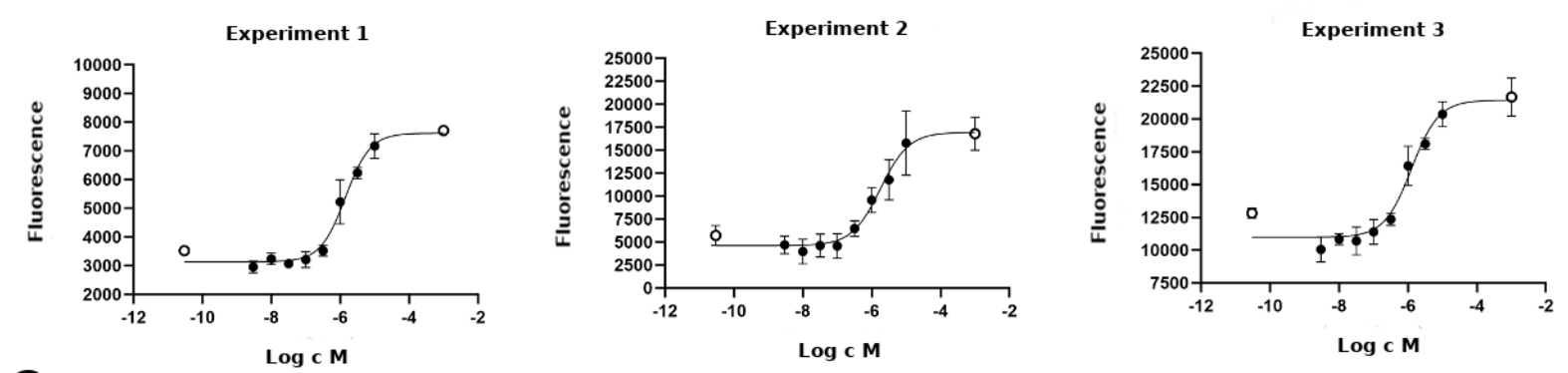

C
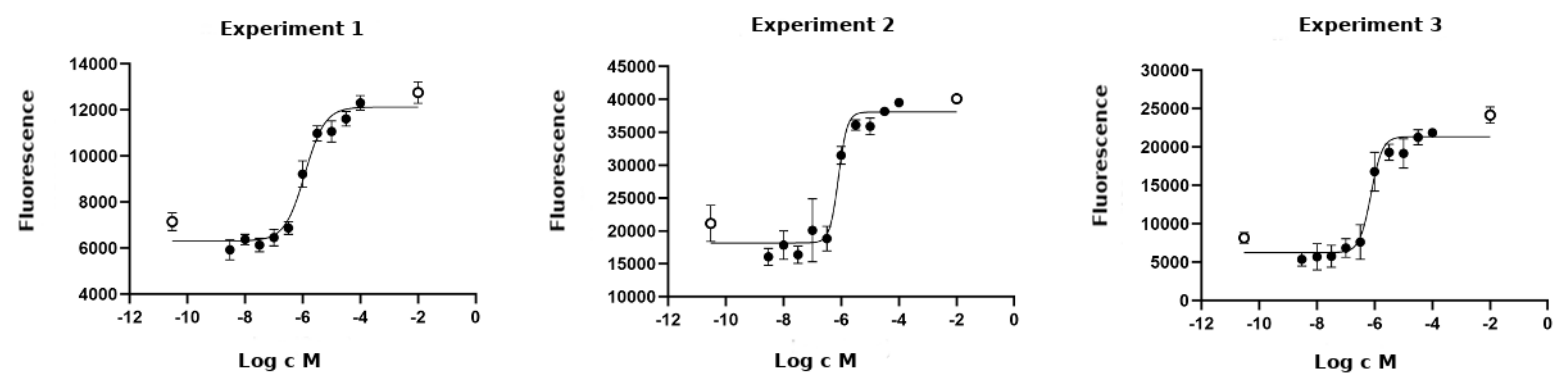

Supplementary figure 4: Potency measurements of the hit compounds for all three repeated experiments. A) ADPr B) BI-78D3 C) BAY 11-7085. All measurements were carried out in quadruplicates using halflogarithimic dilutions starting from $100 \mu \mathrm{M}$ to $0.003 \mu \mathrm{M}$ for each curve. Data reported as mean \pm SD. 


\section{MacroD2}

$\mathbf{A}$
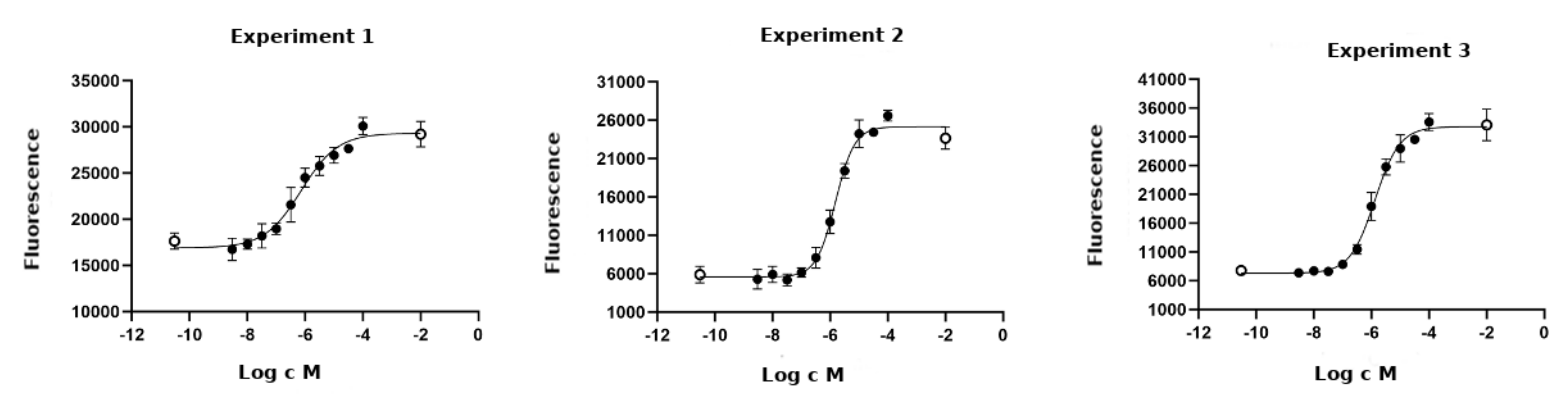

B
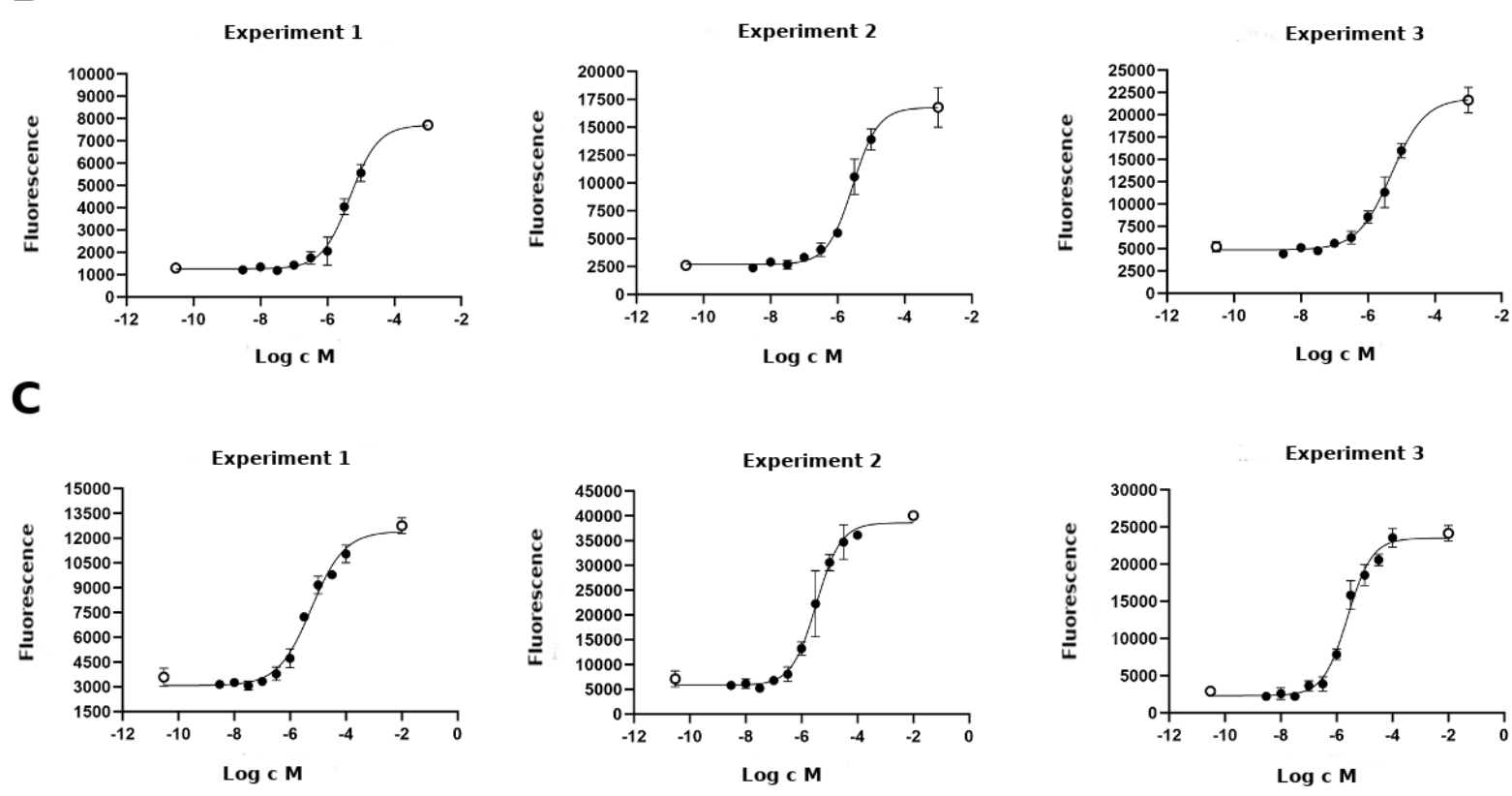

Supplementary figure 5: Potency measurements of the hit compounds for all three repeated experiments. A) ADPr B) BI-78D3 C) BAY 11-7085. All measurements were carried out in quadruplicates using halflogarithimic dilutions starting from $100 \mu \mathrm{M}$ to $0.003 \mu \mathrm{M}$ for each curve. Data reported as mean $\pm \mathrm{SD}$. 
$\mathbf{A}$

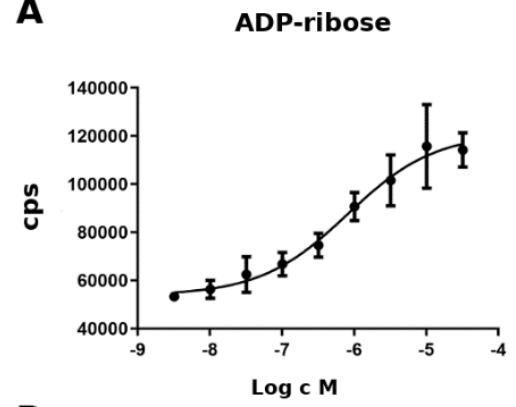

B

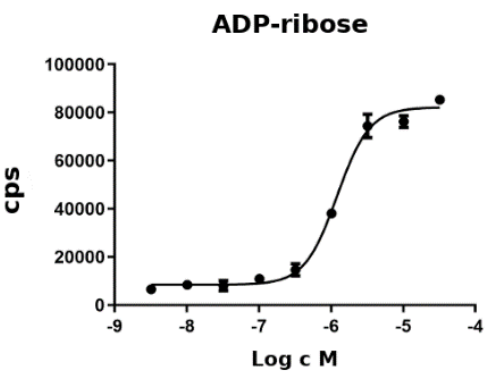

BI-78D3

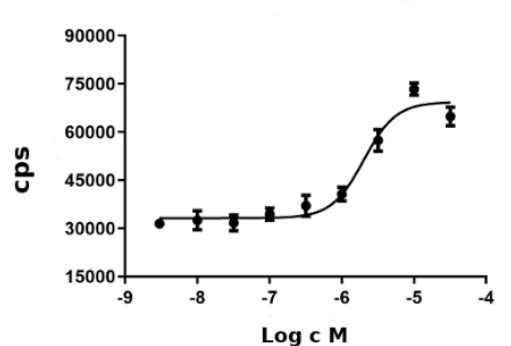

BI-78D3

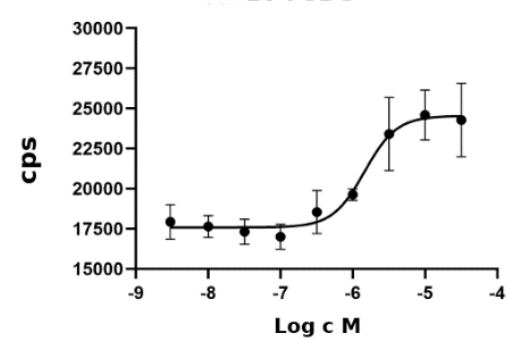

BAY 11-7085

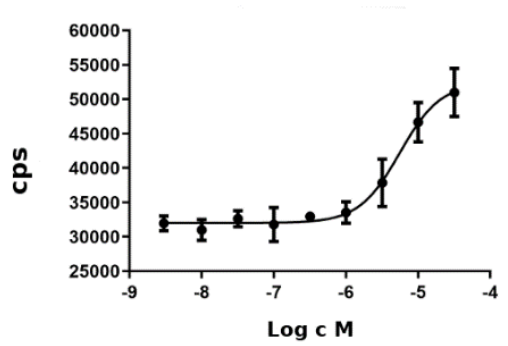

BAY 11-7085

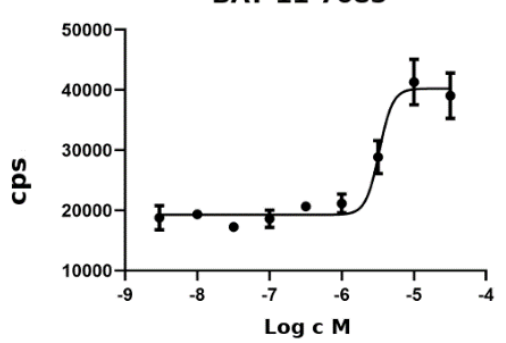

Supplementary figure 6. Potency measurements of the hit compounds utilizing AlphaScreen assay. A) MacroD1 B) MacroD2. All measurements were carried out in quadruplicates using half-logarithimic dilutions starting from $32 \mu \mathrm{M}$ to $0.003 \mu \mathrm{M}$ for each curve. Data reported as mean $\pm \mathrm{SD}$.

A

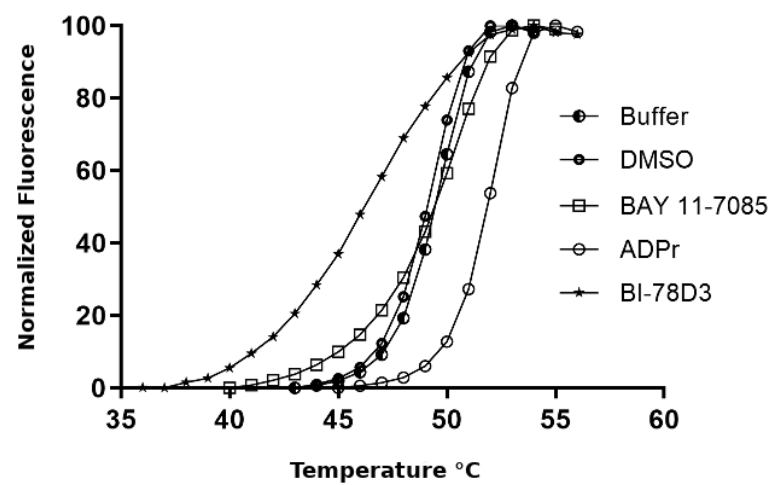

B

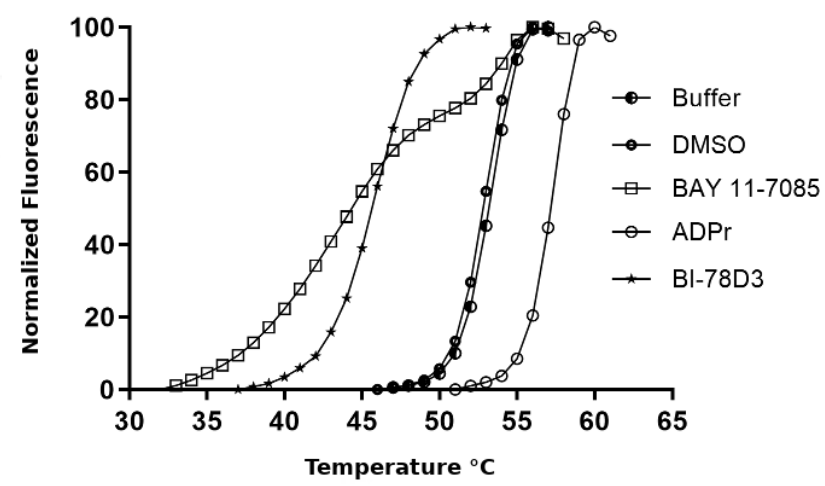

Supplementary figure 7. Thermal shift assay for (A) MacroD1 and (B) MacroD2. Samples were prepared in triplicates. Data points are shown on each curve and means for all replicates of controls and compounds for the macrodomains are fitted to obtain the curves. The assay utilized ADPr and the hit compounds BI-78D3 and BAY 11-7085. 

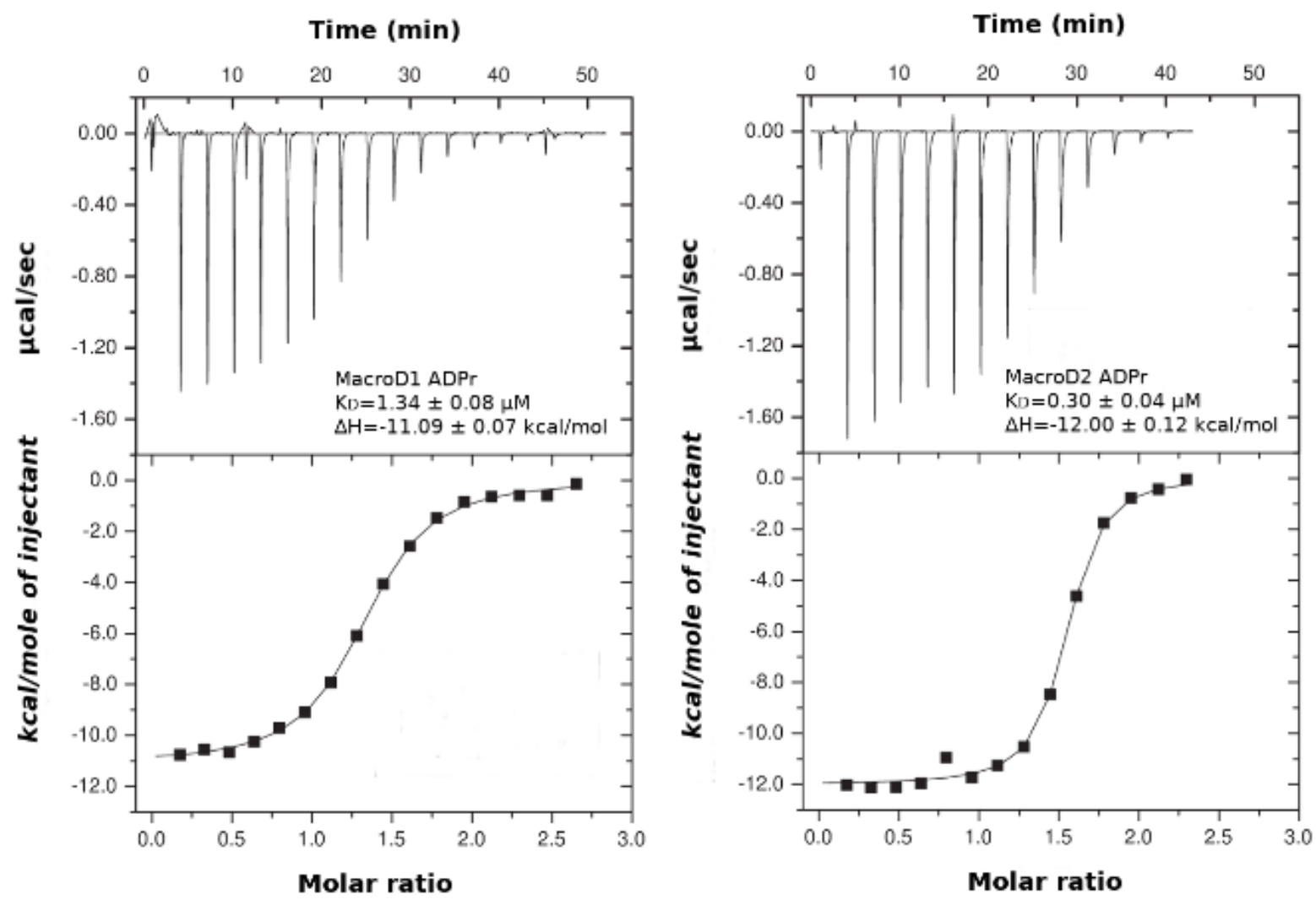

Supplementary figure 8: ADPr binding affinity of the mono-ADPr hydrolases. Isothermal titration calorimetry profile of ADPr titrated with MacroD1 and MacroD2 is depicted at the top. Data points are shown on each curve and the curves are fitted to obtain equilibrium dissociation constant $\mathrm{K}_{\mathrm{D}}$ for the binding of ADPr to each enzyme. The stoichiometry ( $\mathrm{n}$ ) of the interaction was measured to be $1.29 \pm 0.0$ and $1.48 \pm 0.01$ for MacroD1 and MacroD2, respectively. 